\title{
Elastic scattering of quasi-free electrons in strongly asymmetric collisions
}

\author{
M Kuzel, R D DuBoist, R Maier, O Heil $\ddagger$, D H Jakubaßa-Amundsen§, \\ $\mathrm{M}$ W Lucas $\|$ and $\mathrm{K} O \mathrm{O}$ Groeneveld \\ Institut für Kernphysik der J W Goethe Universitāt, August Euler Straße 6, 60486 Frankfurt \\ a. M., Germany
}

Received 25 October 1993

\begin{abstract}
A systematic study of the doubly differential cross sections for the electron emission occurring in fast strongly asymmetric collisions is presented. Protons and atomic hydrogen $(0.5 \mathrm{MeV})$ were collided with the heavy targets krypton and xenon under single collision conditions. The ejected electrons were observed at laboratory angles in the range $0^{\circ} \leqslant \theta_{f} \leqslant 180^{\circ}$ and with energies $E_{f}$ from 25 to $1400 \mathrm{eV}$.

In addition to the familiar strong ejection in the forward direction $\left(\theta_{f} \approx 0^{\circ}\right)$ arising from capture and loss to continuum states there is pronounced emission in the backward hemisphere for the case of atomic hydrogen projectiles. This is accompanied by large variations in the angular dependent singly differential cross section and in the energy and width of the electron loss peak as measured at particular angles. These fluctuations in the ability of a heavy target to ionize the structured projectile are a manifestation of a RamsauerTownsend effect and can be understood within the framework of quasi-free electron scattering by the target potential. We give a relativistic quantum mechanical model based on the electron impact approximation which shows good agreement with experiment for these strongly asymmetric collision systems.
\end{abstract}

\section{Introduction}

The interaction of structured projectiles with heavy target atoms can lead to excitation and ionization of both projectile and target, as well as charge exchange. Here we wish to focus attention on the ionization of fast atomic hydrogen by the heavy targets krypton and xenon. A number of similar studies are referenced in Hartley and Walters (1987), Kövér et al (1988), DuBois and Manson (1990) and Kuzel et al (1992) but theoretical analysis of the problem is made difficult by the inability of almost all experiments to separate the singly inelastic (s1) from the doubly inelastic (DI) contributions to the measured signal (Bates and Griffing 1954). Montenegro et al (1992) have attacked this problem experimentally and there have also been two recent theoretical attempts (Jakubaßa-Amundsen 1992, Wang et al 1992) to incorporate the DI channel into the † Permanent address: Pacific Northwest Laboratory, PO Box 999, Richland, WA 99352, USA.
† Present address: Lahmeyer International, 60528 Frankfurt, Germany.
§ Permanent address: Sektion Physik der Universität München, Am Coulombwall 1, 85748 Garching,
Germany.
If Permanent address: School of Mathematical and Physical Sciences, University of Sussex, Brighton BN1 9QH, UK. 
theory for backward emission angles. These have shown considerable success for fast $\mathrm{H}^{0}$ and $\mathrm{He}^{+}$impacting light targets but difficulties remain in the case of argon where calculations of the singly inelastic channel alone can overpredict the experimental signal. Adding a contribution from the $\mathrm{D}$ channel exacerbates the problem, particularly for $\mathrm{He}^{+}$projectiles, even if care is taken to restrict target ionization to the valence electrons only (Jakubaßa-Amundsen 1993). We have not specifically attempted to resolve this difficulty with the SI and DI contributions here but rather sought to gain some understanding of the systematics involved. By using even heavier targets we seek to determine whether the theoretical overprediction simply becomes worse as the number of target electrons increases and to what extent shell effects are important.

The simplest structured projectile $\mathrm{H}^{0}(0.5 \mathrm{MeV})$, predominantly in the ground state, was collided with $\mathrm{Kr}$ and $\mathrm{Xe}$ and the emitted electrons observed over the complete angular range $0^{\circ} \leqslant \theta_{f} \leqslant 180^{\circ}$ and an energy range from $25 \mathrm{eV}$ to $1400 \mathrm{eV}$. Because we wished to direct our major interest upon the ionization of the projectile we repeated all measurements with an $\mathrm{H}^{+}$beam of the same energy. The doubly and singly differential cross sections for both projectiles are compared. We assume that the pure target ionization caused by $\mathrm{H}^{0}$ and $\mathrm{H}^{+}$is the same, apart from possible screening effects at the lowest energies, so that the difference in the two signals arises from projectile electrons alone. It is known that the electrons lost by the projectile give rise to the broad 'electron loss peak' (Wilson and Toburen 1973, Burch et al 1973, Drepper and Briggs 1976) which reflects the Compton profile of their initial bound state. For very small emission angles $\theta_{f} \approx 0^{\circ}$ relative to the projectile motion it has a cusp shape because of the final-state interaction with the projectile nucleus. The intensity of the electron loss peak decreases rapidly with larger emission angles due to the need for higher momentum transfer to the electron. However, our data show large variations in the angular dependence of the projectile ionization cross sections in the backward hemisphere $\left(\theta_{f}>90^{\circ}\right)$. These variations are similar to those found for free electron elastic scattering from heavy targets (Ramsauer 1921, Townsend and Bailey 1922, McCarthy et al 1977) but there are significant and interesting differences caused by the initial binding to the proton.

We employed a relativistic prescription of the electron impact approximation (EIA) (Jakubaßa 1980, Jakubaßa-Amundsen 1992) to calculate electron loss cross sections. In the EIA, the final electronic state is described by a target scattering eigenstate such that electron loss is treated in terms of quasielastic scattering of the projectile electron on the target potential. The comparison of our measured absolute doubly differential cross sections for electron emission with the ElA allows a sensitive test of this theoretical model.

\section{Experiment}

The experiments were performed at the $2.5 \mathrm{MV}$ Van de Graaff accelerator at the Institut für Kernphysik of the $\mathrm{J} W$ Goethe University, Frankfurt. We used a primary beam consisting of $1.0 \mathrm{MeV} \mathrm{H}_{2}^{+}$molecules which entered a gas cell (air at $4 \times 10^{-5}$ mbar) after passing through the bending magnet. Some fraction $(\approx 1 \%)$ of these molecules were thereby dissociated into $\mathrm{H}^{+}$and neutral $\mathrm{H}^{0}$ each with an energy $0.5 \mathrm{MeV}$. The fraction of neutral $\mathrm{H}_{2}$ molecules produced is negligible at this energy. At the exit of the cell 


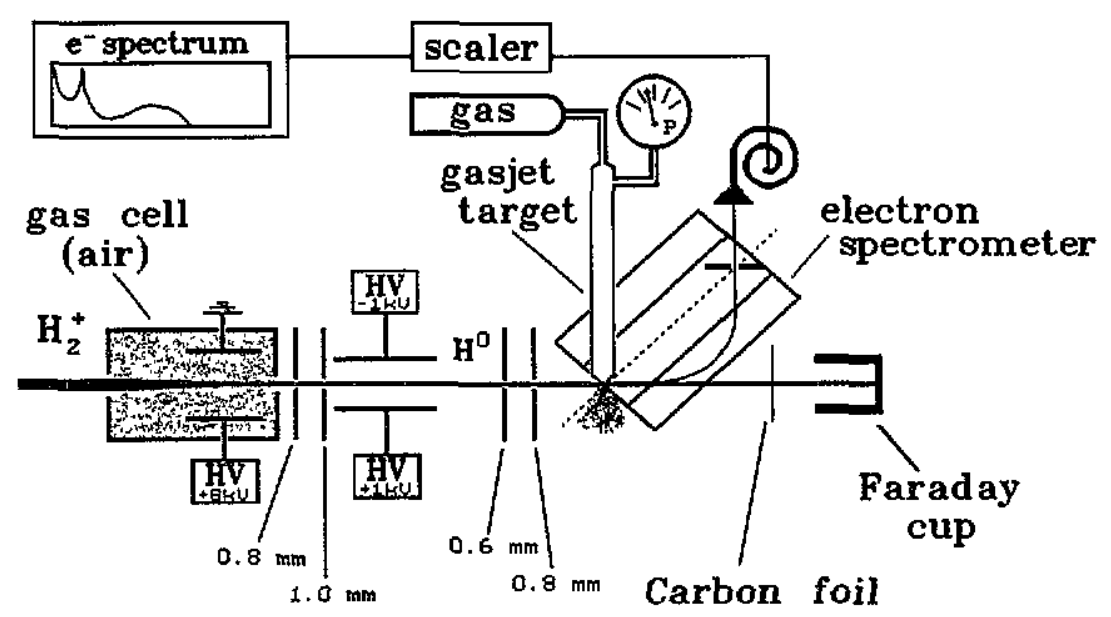

Figure 1. Schematic view of the experimental apparatus.

(figure 1) a strong electric field $\left(\approx 5 \mathrm{kV} \mathrm{cm}^{-1}\right)$ deflected any charged components out of the beam path. A second electric field $0.5 \mathrm{~m}$ in front of the target further reduced the amount of charged beam components, so that finally more than $98 \%$ of the projectiles were neutral and in the ground state (Bethe and Salpeter 1957). Two pairs of apertures collimated the beam to a diameter of $0.6 \mathrm{~mm}$ in the interaction region where it was intersected by a directed gas jet target. The outgoing projectiles were stripped by a thin carbon foil $\left(20 \mu \mathrm{g} \mathrm{cm}^{-2}\right)$ and then collected in a biassed Faraday cup. Foils were checked against pinholes using an electron gun. The gas target density was controlled by a capacitance manometer driving a magnetic valve. To monitor the stability of the target density a surface barrier detector recorded projectiles which were Rutherford scattered at $10^{\circ}$ relative to the beam axis.

The apertures of the electrostatic cylindrical mirror electron spectrometer (Bernardi et al 1988) were chosen to combine good energy and angular resolution $(\Delta E / E=3.14 \%$, $\Delta \theta=1.67^{\circ}$ ) with high transmission. The spectrometer can be rotated under vacuum to analyse electrons emitted at angles $0^{\circ} \leqslant \theta_{f} \leqslant 180^{\circ}$, except for two instrumentally shadowed areas between $63^{\circ}$ and $87^{\circ}$ and between $93^{\circ}$ and $117^{\circ}$. A set of three mutually perpendicular Helmholtz coils reduced the Earth's magnetic field to about $3 \%$ of its normal value. The electron spectra were taken by increasing the spectrometer voltage in steps of $2 \mathrm{~V}(\approx 3.25 \mathrm{eV})$ and normalizing each channel to a fixed accumulated charge in the Faraday cup.

We also measured spectra for proton impact on $\mathrm{Kr}$, where no projectile ionization can occur, to determine the magnitude of the 'pure' target ionization which contributes to our electron spectra in the case of hydrogen impact. To obtain absolute values for the doubly differential cross sections $\left(\mathrm{d}^{2} \sigma / \mathrm{d} E_{f} \mathrm{~d} \Omega_{f}\right.$ ) we measured spectra for the collision system $\mathrm{H}^{+}(0.5 \mathrm{MeV}) \rightarrow \mathrm{Ar}$ and normalized to the absolute data given by Rudd $e t$ al (1976). In this way we could obtain the transmission and detector efficiency for the spectrometer system. To deduce the number density of the $\mathrm{Kr}$ and $\mathrm{Xe}$ targets relative to Ar we made use of the Rutherford scattered projectiles which were detected during the experiment. The absolute uncertainties in our doubly differential cross sections (DDCS) due to the normalization method and statistical errors are $80 \%$ for both the krypton and xenon target. The relative errors between individual electron spectra and in the singly differential cross sections $\left(\mathrm{d} \sigma / \mathrm{d} \Omega_{f}\right)$ are less than $20 \%$. 


\section{Theory}

The basic concept of the electron impact approximation is an exact treatment of the final-state interaction between the ejected projectile electron and the multielectron target in the elastic channel. This is accomplished by choosing a target scattering eigenstate for the electronic final state, at the expense of neglecting the influence of the projectile core field. For the strongly asymmetric collision systems considered here such a prescription is reasonable for electrons emitted at angles $\theta_{f} \geqslant 20^{\circ}$ with respect to the beam direction.

The theoretical model is a relativistic extension of the EIA theory developed for light targets (Jakubaßa-Amundsen 1992) and we shall only briefly discuss the two contributions to the electron loss cross section, the singly inelastic and the doubly inelastic parts.

\subsection{Singly inelastic (SI) contribution}

For hydrogen impact, the dominant contribution to electron loss originates from collisions which leave the target in its ground state. The amplitude for the transition of the projectile electron from the initial bound state $\psi_{i}^{\mathrm{P}}$ to a target scattering state $\psi_{f}^{\mathrm{T}}$ is, in lowest order in the electron-projectile core coupling, given by (in atomic units)

$$
a_{f i}^{\mathrm{SI}}=-\mathrm{i} \int \mathrm{d} t\left\langle\psi_{f}^{\mathrm{T}}\left|V_{\mathrm{T}}\right| \psi_{i}^{\mathrm{P}}\right\rangle
$$

where $V_{\mathrm{T}}$ is the effective electron-target interaction averaged over the ground state configuration $\phi_{i}^{\mathrm{T}}$ of the target electrons. We introduce the relativistic amplitude for elastic scattering of an electron (with momentum $\mathrm{k}$ and scattering angle $\theta$ ) from the target (Landau and Lifschitz 1970)

$f_{\mathrm{c}}(k, \theta)=A+B v \sigma$

$A=\frac{1}{2 \mathrm{i} k}\left\{\sum_{i=1}^{\infty}\left[(l+1)\left(\mathrm{e}^{2 \mathrm{i} \delta_{-l-1}}-1\right)+l\left(\mathrm{e}^{2 \mathrm{i} \delta_{i}}-1\right)\right] P_{l}(\cos \theta)+\left(\mathrm{e}^{2 \mathrm{j} \delta_{-l}}-1\right)\right\}$

$B=\frac{1}{2 k} \sum_{l=1}^{\infty}\left(\mathrm{e}^{2 \mathrm{i} \delta_{-i-1}}-\mathrm{e}^{2 \mathrm{i} \delta_{l}}\right) P_{j}^{\prime}(\cos \theta)$

where $v$ is a unit vector perpendicular to the scattering plane, $\sigma$ is the spin vector of the electron, $\delta_{x}$ (with $x=l$ for $x>0$ and $x=-l-1$ for $x<0$ ) are the phaseshifts and $P_{t}$ and $P$ the Legendre polynomials and associated functions, respectively. The doubly differential cross section for electrons ejected with energy $E_{f}=k_{f}^{2} / 2$ into the solid angle $\mathrm{d} \Omega_{f}$ (in the target reference frame) can be written in the following way

$$
\frac{\mathrm{d}^{2} \sigma^{\mathrm{sl}}}{\mathrm{d} E_{f} \mathrm{~d} \Omega_{f}}=\frac{k_{f}}{v} \int \mathrm{d} q\left|\varphi_{1}^{\mathrm{P}}(q-v)\right|^{2}\left|f_{\mathrm{e}}\left(q, k_{f}\right)\right|^{2} \delta\left(E_{f}-\varepsilon_{i}^{\mathrm{P}}+\frac{v^{2}}{2}-q v\right)
$$

In this expression $\varepsilon_{i}^{\mathrm{P}}$ is the initial-state energy and $v$ the collision velocity. Hence, electron loss is described by quasielastic electron scattering from the target folded with the momentum distribution $\varphi_{i}^{p}$ of the initial bound state. Quasielasticity is accounted 
for by means of the on-shell prescription (Hartley and Walters 1987)

$$
f_{\mathrm{e}}\left(\boldsymbol{q}, \boldsymbol{k}_{f}\right) \approx f_{e}(k, \theta) \quad k=\max \left(q, k_{f}\right) \quad \sin \frac{\theta}{2}=\frac{\left|\boldsymbol{q}-\boldsymbol{k}_{f}\right|}{2 k} .
$$

From (3.3) it is readily seen that the cross section is peaked near $q=v$, i.e. near an energy

$$
E_{f}=\varepsilon_{i}^{P}+v^{2} / 2
$$

The shape of this electron loss peak is basically determined by the momentum distribution of the initial state, but it is also influenced by the rapid variation of the elastic scattering amplitude (Jakubaßa-Amundsen 1993).

For unpolarized electrons an average over the spin directions has to be performed such that the elastic scattering cross section reduces to

$$
\frac{\mathrm{d} \sigma_{\mathrm{e}}}{\mathrm{d} \Omega}=\left|f_{\mathrm{e}}(k, \theta)\right|^{2}=|A|^{2}+|B|^{2} .
$$

The si cross section then acquires the final form

$$
\frac{\mathrm{d}^{2} \sigma^{\mathrm{Sl}}}{\mathrm{d} E_{f} \mathrm{~d} \Omega_{f}}=\frac{2 k_{f}}{v^{2}} \int_{q_{\min ,}}^{\infty} q \mathrm{~d} q \int_{0}^{\pi} \mathrm{d} \varphi_{q}\left|\varphi_{i}^{\mathrm{P}}(\boldsymbol{q}-\boldsymbol{v})\right|^{2}\left[\left|A\left(\boldsymbol{q}, \boldsymbol{k}_{f}\right)\right|^{2}+\left|B\left(\boldsymbol{q}, \boldsymbol{k}_{f}\right)\right|^{2}\right]
$$

with $q_{\mathrm{min}, \mathrm{c}}=\left(E_{f}-\varepsilon_{i}^{\mathrm{P}}+v^{2} / 2\right) / v$. The peaking approximation applied earlier for helium targets (Jakubaßa-Amundsen 1992) would not be sufficiently accurate at backward emission angles for very heavy targets because the scattering cross section (3.6) varies strongly with energy and angle. Therefore, (3.7) is used without additional approximations, a fairly simple procedure in the case of this sI contribution since only a single momentum integral is involved.

\subsection{Doubly inelastic (DI) contribution}

At low electron ejection energies there is a significant contribution to electron loss arising from the simultaneous ionization of projectile and target. In the second-order Born approximation the transition amplitude for this DI process is given by

$a_{f i}^{\mathrm{D} 1}=-\mathrm{i} \int \mathrm{d} t\left\langle\phi_{f}^{\mathrm{T}} \psi_{f}^{\mathrm{T}}\left|V_{\mathrm{ee}}\right| \phi_{i}^{\mathrm{T}} \psi_{i}^{\mathrm{P}}\right\rangle-\mathrm{i} \int \mathrm{d} t\left\langle\phi_{f}^{\mathrm{T}} \psi_{f}^{\mathrm{T}}\left|V_{\mathrm{Pe}} G_{0} V_{\mathrm{T}}\right| \phi_{i}^{\mathrm{T}} \psi_{i}^{\mathrm{P}}\right\rangle$

where $\phi_{i}^{\top}$ and $\phi_{f}^{\top}$ respectively denote the ground state and final state configuration of the target electrons. The first term, where electron loss is mediated by the projectile electron-target electron coupling $V_{\mathrm{ce}}$, is the 'coherent' projectile-target ionization. The second term describes the 'incoherent' projectile-target ionization. Here the projectile electron is ejected by means of $V_{\mathrm{T}}$, while target ionization is caused by the interaction $V_{\mathrm{Pc}}$ with the projectile core field. In the intermediate state the projectile electron propagates in the target potential, i.e. $G_{0 f}=\left(\mathrm{i} \partial_{t}-H_{\mathrm{T}}-T_{\mathrm{c}}-V_{\mathrm{T}}+\mathrm{i} \varepsilon\right)^{-1}$ where $T_{\mathrm{c}}$ is the kinetic energy of the projectile electron and $H_{\mathrm{T}}$ the electronic Hamiltonian of the target atom. For emission angles above $30^{\circ}$ a high momentum transfer to the projectile electron is required and hence an additional coherent contribution to the second order term (Jakubaßa-Amundsen 1992) is neglected. For the same reason the first order coherent projectile-target ionization is also small. Restricting ourselves to the contribution from 
the incoherent projectile-target ionization and considering only ionization of the valence electrons, which is much more important than excitation to bound states or ionization of the inner electrons, the doubly differential DI cross section for a one-electron projectile is found from

$$
\begin{aligned}
\frac{\mathrm{d}^{2} \sigma^{\mathrm{DI}}}{\mathrm{d} E_{f} \mathrm{~d} \Omega_{f}}=\frac{k_{f} Z_{\mathrm{p}}^{2}}{4 \pi^{4} v} \sum_{n} \int \mathrm{d} x_{\mathrm{T}} \int \mathrm{d} q \delta\left(\Delta E_{f n}^{\mathrm{T}}+E_{f}-\varepsilon_{i}^{P}+v^{2} / 2-q v\right) \\
\times\left\{\left|\int \frac{\mathrm{d} s}{s^{2}} \varphi_{i}^{\mathrm{P}}(q-v-s) A\left(q-s, k_{f}\right) F_{\mathrm{T}}(s)\right|^{2}\right. \\
\left.+\left|\int \frac{\mathrm{d} s}{s^{2}} \varphi_{i}^{\mathrm{P}}(q-v-s) B\left(q-s, k_{f}\right) F_{\mathrm{T}}(s)\right|^{2}\right\} \\
F_{\mathrm{T}}(s)=\frac{1}{\Delta E_{f n}^{\mathrm{T}}-s v+\mathrm{i} \varepsilon}\left\langle\varphi_{f}^{\mathrm{T}}\left|\mathrm{e}^{s s \mathrm{~T}}\right| \varphi_{n}^{\mathrm{T}}\right\rangle
\end{aligned}
$$

with $Z_{\mathrm{P}}$ the projectile nuclear charge and $A$ and $B$ from (3.2). The state $\varphi_{n}^{\top}$ is a single particle target valence state and the sum runs over all valence states $n . \Delta E_{f_{n}}^{\top}$ is the target excitation energy and $\varphi_{f}^{\mathrm{T}}$ a target continuum state with momentum $x_{\mathrm{T}}, \boldsymbol{r}_{\mathrm{T}}$ denoting the electron coordinate in the target reference frame. In (3.9) we have not made the closure approximation used in previous work (Kuzel et al 1992). Such a closure approximation would imply an equal participation of all target electrons to the excitation process in the case of large momentum transfer and would strongly overestimate the cross section for very heavy targets.

Just as in the case of the sI cross section (3.3), the DI cross section is governed by the quasielastic electron scattering from the target folded with the initial state momentum distribution. In addition one has to fold with the target excitation function $F_{\mathrm{T}}(s)$ and this makes (3.9) sensitive to the phase of the scattering amplitude $f_{\mathrm{e}}$. From the Fourier transformed projectile field, proportional to $1 / s^{2}$, and from $\varphi_{1}^{\mathrm{P}}(q-v-s)$ one can infer that the DI cross section is also peaked near $q=v$, but the corresponding energy

$$
E_{f} \approx \varepsilon_{i}^{\mathrm{P}}+\frac{v^{2}}{2}-\overline{\Delta E^{\mathrm{T}}}
$$

is smaller. Here $\overline{\Delta E}$ T is a mean target excitation energy. This implies that the electron loss peak is shifted to a smaller energy when the DI contribution is included.

For $\mathrm{Kr}$ and $\mathrm{Xe}$ targets the spin-flip scattering amplitude $B$ turns out to be small and can safely be neglected. Unfortunately, (3.9) involves two momentum integrals and its exact evaluation is computationally difficult. For this DI contribution we did resort to the earlier peaking approximation (Jakubaßa-Amundsen 1992) which relies on the fact that the $s$ integrals in (3.9) are dominated by the functional dependence of the bound state function $\varphi_{i}^{\mathrm{P}}$. The DI cross section then reduces to

$$
\begin{aligned}
\frac{\mathrm{d}^{2} \sigma^{\mathrm{DI}}}{\mathrm{d} E_{f} \mathrm{~d} \Omega_{f}}=\frac{4 Z_{\mathrm{P}}^{7} k_{f}}{\pi^{3} v^{2}} \sum_{n} \int_{0}^{\infty} \chi_{\mathrm{T}}^{2} \mathrm{~d} x_{\mathrm{T}} \int_{q_{\min , l}}^{\infty} q \mathrm{~d} q \bar{G}_{n}\left(x_{\mathrm{T}}, p\right) \\
\quad \times\left|\int_{-\infty}^{\infty} \mathrm{d} s_{z} \frac{1}{\Delta E_{f_{n}}^{\mathrm{T}}-s_{z} v+\mathrm{i} \varepsilon} A\left(\left(q_{z}-s_{z}\right) e_{z}, k_{f}\right) x_{i}\left(s_{z}, q\right)\right|^{2}
\end{aligned}
$$




$$
\begin{aligned}
& \bar{G}_{n}\left(\varkappa_{\mathrm{T}}, p\right)=\int \mathrm{d} \Omega_{x}\left|\left\langle\varphi_{f}^{\mathrm{T}}\left|\mathrm{e}^{\mathrm{i} p r \mathrm{~T}}\right| \varphi_{n}^{\mathrm{T}}\right\rangle\right|^{2} \\
& I_{i}\left(s_{z}, q\right)=2^{-3 / 2} Z_{\mathrm{P}}^{-5 / 2} \int \mathrm{d} s_{\perp} \frac{1}{s^{2}} \varphi_{i}^{\mathrm{P}}(q-s-v)
\end{aligned}
$$

where $q_{\min , i}=\Delta E_{f n}^{\mathrm{T}} / v+q_{\min , e}, p=q_{\perp}+\left(\Delta E_{f n}^{\mathrm{T}} / v\right) e_{z}$ and $\Omega_{x}$ is the angular part of $x_{\mathrm{T}}$. The $z$-axis $e_{z}$ is taken along the beam direction and $q_{\perp}$ and $s_{\perp}$ denote the components perpendicular to $e_{z}$. The peaking approximation improves as $\varphi_{i}^{\mathrm{P}}$ becomes more strongly peaked, i.e. the lighter the projectile. Strictly speaking it is, however, rather poor when the scattering amplitude (3.2) is a strongly varying function of electron momentum. We have found that a tentative application to the si cross section (3.7) would lead to a drastic overprediction of the Ramsauer-Townsend effect. Although the peaking approximation applied in (3.11) is not as severe as it would be if used in (3.7) we recognise the limited accuracy of this DI contribution at backward angles for heavy targets.

\subsection{Numerical details}

In order to determine the relativistic phaseshifts $\delta_{x}$ the radial Dirac equation for the large $(F=r f)$ and small $(G=r g)$ component of the target scattering eigenstate has to be solved

$$
\begin{aligned}
& \frac{\mathrm{d} F}{\mathrm{~d} r}+\frac{x}{r} F-\frac{1}{c}\left(E+m c^{2}-V_{\mathrm{T}}\right) G=0 \\
& \frac{\mathrm{d} G}{\mathrm{~d} r}-\frac{x}{r} G+\frac{1}{c}\left(E-m c^{2}-V_{\mathrm{T}}\right) F=0
\end{aligned}
$$

subject to the initial conditions in $r \rightarrow 0$ :

$$
\left.\begin{array}{l}
F=r^{x+1} \\
G=\frac{2 x+1}{E+m c^{2}} c r^{*}
\end{array}\right\} \text { for } x>0
$$

where $E=\left(k^{2} c^{2}+m^{2} c^{4}\right)^{1 / 2}$ is the total energy.

As in the non-relativistic case the phaseshifts are extracted from the behaviour of the radial wavefunction for large $r$

$$
F \rightarrow \frac{\sqrt{E+m c^{2}}}{\sqrt{2 E}} \frac{1}{k} \sin \left(k r-l \frac{\pi}{2}+\delta_{x}\right) .
$$

The effective target field $V_{\mathrm{T}}$ is composed of the relativistic static potential and the polarization field. For krypton we have used the analytic fit to the Hartree-Fock 
potential from Strand and Bonham (1964), such that

$$
\begin{aligned}
& V_{\mathrm{T}}^{\mathrm{KT}}(r)=-\frac{Z_{\mathrm{T}}}{r} \sum_{i}\left(a_{i} \mathrm{e}^{-b_{r} r}+\alpha_{i} r \mathrm{e}^{-\beta_{r} r}\right)-\frac{\alpha r^{2}}{2\left(r^{2}+d^{2}\right)^{3}} \\
& d=\frac{3}{8 \Delta} \begin{cases}k_{0} & k \leqslant k_{0} \\
k & k>k_{0}\end{cases}
\end{aligned}
$$

where $Z_{\mathrm{T}}$ is the target nuclear charge and $a_{i}, b_{i}, \alpha_{i}$ and $\beta_{i}$ are the fit parameters. For the polarization field, the dipole polarizability is $\alpha=16.75$ (Miller and Bederson 1977), the first ionization threshold is $\Delta=0.515$ and the low-energy cut-off parameter $k_{0}$ has been set to 1.4 in order to obtain phaseshifts close to the ones from ab-initio calculations (Sin Fai Lam 1982, McEachran and Stauffer 1984). (All parameters are in atomic units.)

For xenon we have found that the exact phaseshifts (Sin Fai Lam 1982, Sienkiewicz and Baylis 1991) are best reproduced by the following combination of two potentials

$$
V_{\mathrm{T}}^{\mathrm{X}_{\mathrm{e}}}(r)= \begin{cases}\frac{Z_{\mathrm{T}}}{Z_{\mathrm{T}}-1}\left(V_{\mathrm{A}}(r)+\frac{1}{r}\right) & r \leqslant r_{0} \\ -\frac{Z_{\mathrm{T}}}{r} \sum_{i} a_{t} \mathrm{e}^{-b_{i} r}-\frac{\alpha r^{2}}{2\left(r^{2}+d^{2}\right)^{3}} & r>r_{0}\end{cases}
$$

where $r_{0} \sim 3$ is the point of intersection. $V_{\mathrm{A}}(r)$ is the optimized one-electron potential for $\mathrm{Xe}^{+}$(with a $-1 / r$ tail) from Aashamar et al (1978) which includes the polarization effects. Because the tail-corrected potential appropriate for neutral Xe becomes inaccurate at large $r$ we have used the exponential fit to the static potential from Cox and Bonham (1967) plus a polarization field with $\alpha=27.28, \Delta=0.446$ and $k_{0}=1.5$.

For the evaluation of the scattering amplitude for $\mathrm{Kr}$ and $\mathrm{Xe}$ we have found it sufficient to include phaseshifts up to $l=20$. It turned out that relativistic effects are only important for the lowest angular momenta $(l \leqslant 3)$, so that for $l \geqslant 4$ we have restricted ourselves to solving the Dirac equation only for positive $x$ and to using $\delta_{-l-1}=\delta_{l}$ in that case.

In the DI cross section (3.11) we have described the target valence electrons from the filled $s$ and $p$ subshells by a common hydrogenic is wavefunction with a Slaterscreened effective charge $Z_{\text {eff }}\left(Z_{\text {eff }}=2.06\right.$ for $\mathrm{Kr}$ and 1.65 for $\left.\mathrm{Xe}\right)$. The corresponding binding energy is set equal to $\Delta$ such that $\Delta E_{f n}^{\top}=\Delta+\chi_{T}^{2} / 2$ and the sum over $n$ is replaced by the number $N_{\mathrm{v}}=8$ of valence electrons. We have tested this approximation for $\mathrm{Xe}$ by using Roothaan Hartree-Fock wavefunctions (Clementi and Roetti 1974) for the s and $p$ valence states with the respective experimental binding energies. These functions are no longer orthogonal to the Coulomb waves (calculated with $Z_{\text {eff }}$ ) used for the final states $\varphi_{f}^{\mathrm{T}}$, hence we took plane waves for $\varphi_{f}^{\mathrm{T}}$ in the comparison between the HartreeFock functions and the hydrogenic ones. Our results from using a scaled 1s function multiplied by $N_{\mathrm{v}}$ differ from the cross section obtained by summing the s and p state contributions, calculated with the Hartree-Fock functions, by only about $5 \%$. The success of the scaled hydrogenic function is of course related to the fact that one has filled valence subshells and that (3.11) implies an integration over all final target continuum states $\varphi_{f}^{\mathrm{T}}$. Consequently the details of the initial state momentum distributions are of minor importance. 


\section{Results and discussion}

The measured electron spectra contain contributions from both projectile and target electron emission. There is no pronounced structure in the angular distribution of the target ionization except for the 'electron capture to continuum' peak at $\theta_{f} \approx 0^{\circ}, E_{c u s p}=$ $E_{\mathrm{P}} m_{\mathrm{c}} / m_{\mathrm{P}}$ (Crooks and Rudd 1970) and the 'binary encounter peak' (BEP) near $E_{\mathrm{BEP}}=$ $4 E_{\text {cusp }} \cos ^{2} \theta_{f}$ in the forward hemisphere $\left(\theta_{f}<90^{\circ}\right)$. As can be seen for example in figure $2(b)$ the 'pure' target ionization for proton impact is largest for very low electron energies $E_{f} \rightarrow 0$ but is always at least one order of magnitude smaller than the projectile ionization, for electron energies greater than $150 \mathrm{eV}$, so its contribution to the measured spectra is rather insignificant making an explicit subtraction unnecessary.

When the projectile carries an electron $\left(\mathrm{H}^{0}\right)$ a peak produced by those electrons lost by the projectile dominates the spectra near $E_{\text {cusp }}$ (figure $2(a)$ ). At $\theta_{f} \approx 0^{\circ}$ the electrons are lost into the projectile continuum and thus the peak remains cusp shaped. For
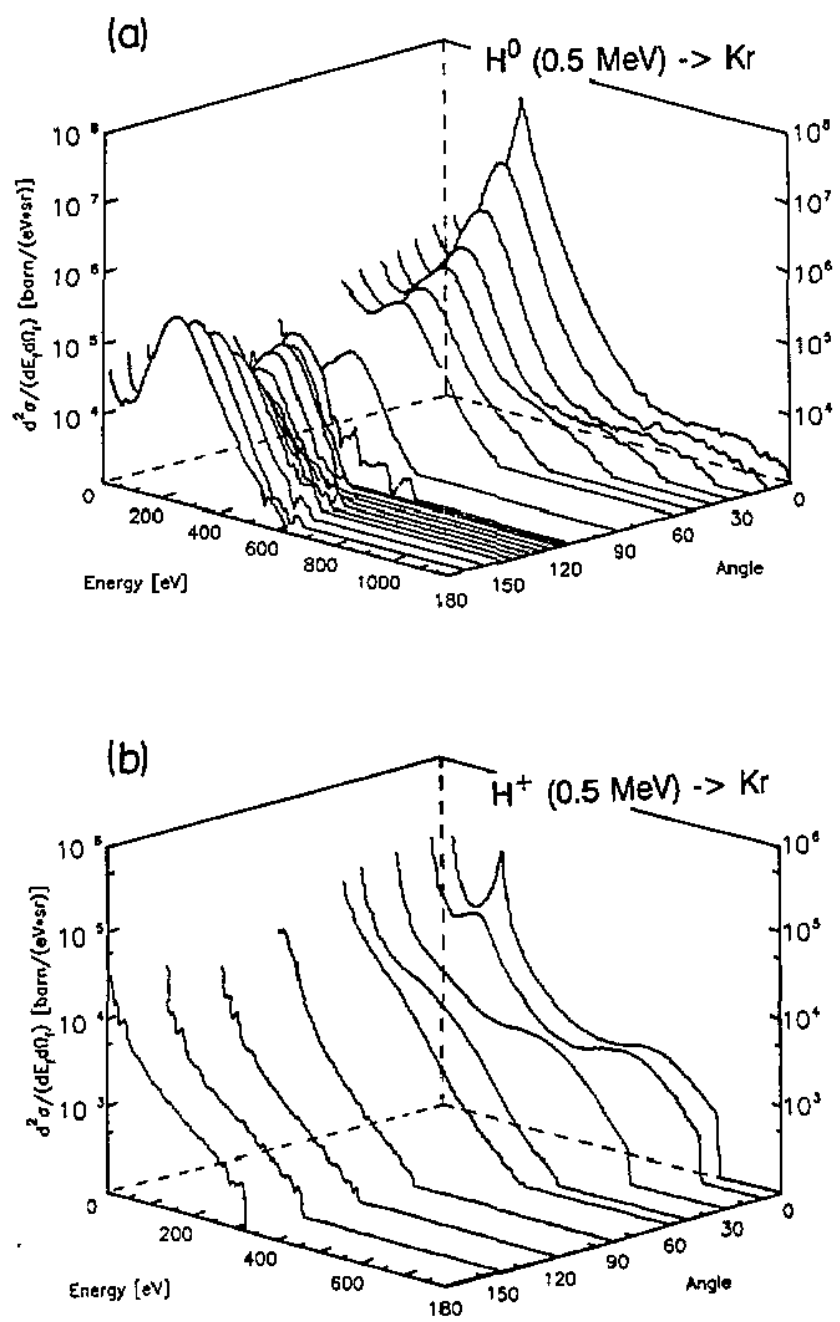

Figure 2. Absolute doubly differential cross sections for $(a) \mathrm{H}^{\circ}$ and $(b) \mathrm{H}^{+}$impact on $\mathrm{Kr}$. Here and in subsequent figures the impact energy is $0.5 \mathrm{MeV}$. 
larger, but still forward, emission angles the intensity of this ELP decreases with increasing emission angle eventually having fallen by about two orders of magnitude at $\theta_{f} \approx 60^{\circ}$; also the peak becomes rounded. In the backward hemisphere this tendency is reversed and the loss peak grows again reaching a maximum at $180^{\circ}$. However this growth is not necessarily smooth as in the case of Ar (Kuzel et al 1992) but can show maxima and minima. For $\mathrm{Kr}$ there is an important maximum near $\theta_{f} \approx 120^{\circ}$ and a minimum at $135^{\circ}$. A second minimum predicted by our theory to be near $80^{\circ}$ is unfortunately not accessible to our spectrometer. In figure 3 we have selected four particular



Figure 3. Doubly differential cross sections for $\mathrm{H}^{\circ} \rightarrow \mathrm{Kr}$, electron emission angles $\theta_{f}=40^{\circ}$, $120^{\circ}, 140^{\circ}$ and $150^{\circ} ; \mathrm{O}$, experiment, singles data; - , theory, EIA, SI; - . - theory, EIA, SI + DI.

ejection angles for the $\mathrm{H}^{0} \rightarrow \mathrm{Kr}$ system in order to compare our measured and theoretical DDCS. The shape of the energy distributions is well reproduced at all angles except for the lowest electron energies where we know the data suffer from an unwanted contribution due to the target ionization. Theory and experiment also compare well on an absolute scale except at the largest angles where the data lie above the theory.

Before discussing the comparison between our theoretical singly differential cross section (SDCs) and experiments we use our theory to calculate the elastic scattering cross section for free electrons by $\mathrm{Kr}$ and test it against the exact calculation of McCarthy $e t$ al (1977) at $300 \mathrm{eV}$ impact energy. From figure $4(a)$ we see that our calculation can reproduce the essential features of the McCarthy result, particularly the RamsauerTownsend minima. Further, there are only slight changes when, instead of $300 \mathrm{eV}$, the electron impact energy is set to $273 \mathrm{eV}$. This corresponds to the mean velocity of the electron carried in by the $\mathrm{H}^{0}$ projectile.

To obtain an experimental yield for electron loss (figure $4(b)$ ) we compute sDCs $\left(\mathrm{d} \sigma / \mathrm{d} \Omega_{f}\right)$ by integrating the doubly differential electron spectra over the energy range 


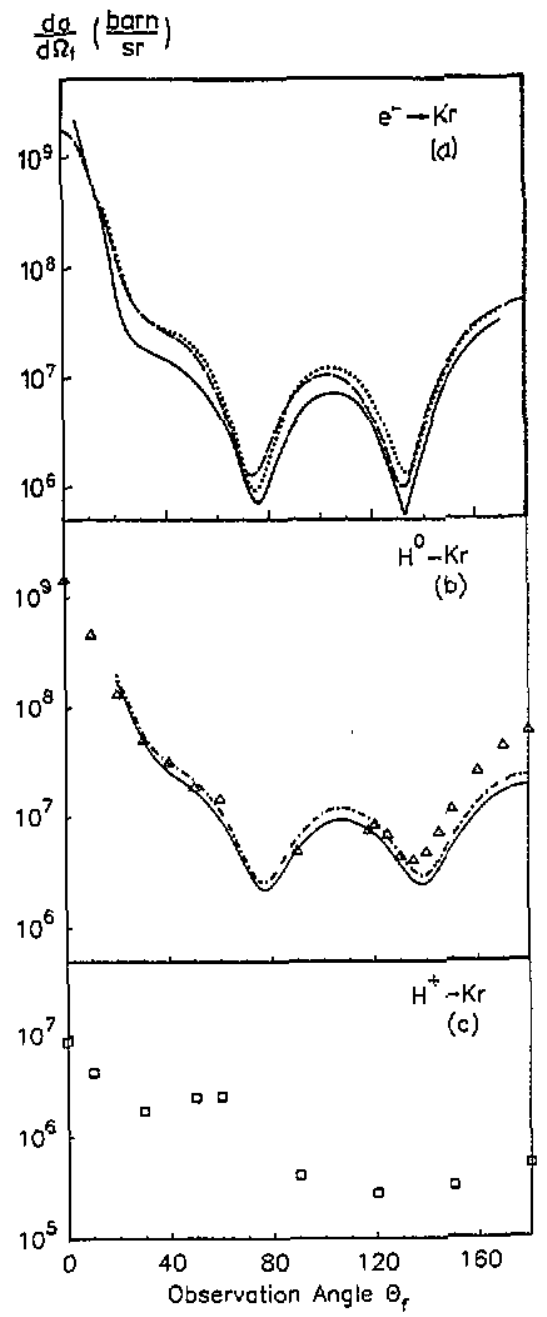

Figure 4. (a) Elastic scattering of free electrons on $\mathrm{Kr}:-$, McCarthy et af (1977) at $300 \mathrm{eV} ;-\cdots$, present theory at $300 \mathrm{eV}$, using model potential; $\cdots$, present theory at $273 \mathrm{eV}$. (b) sDCs for $\mathrm{H}^{0} \rightarrow \mathrm{Kr}: \triangle$, experiment, DDCs integrated over the range $150<E_{f}<400 \mathrm{eV} ;-$, theory, EIA, sI; - . - , theory, EIA, SI + DI. (c) SDCS for $\mathrm{H}^{+} \rightarrow \mathrm{Kr}$, experiment.

$150-400 \mathrm{eV}$. The singly differential cross sections $\left(150 \mathrm{eV}<E_{f}<400 \mathrm{eV}\right)$ for $\mathrm{H}^{+} \rightarrow \mathrm{Kr}$ are shown in figure $4(c)$. Comparison between figures $4(b)$ and $(c)$ shows that the cross sections for electron loss in this peak region are at least one order of magnitude larger than 'pure' target ionization for almost every emission angle. Therefore we can neglect the target electron contribution near the ELP maximum. The unwanted signal in the singly differential cross sections ( $\mathrm{d} \sigma / \mathrm{d} \Omega_{f}$ ) caused by 'pure' target ionization is less than $10 \%$ except around $\theta_{f} \approx 50^{\circ}$ where the BEP is large and shifted into the ELP energy region. Due to this target ionization we will slightly overestimate the experimental values of the cross sections for projectile ionization. In these sDCS the pronounced intensity variations at backward angles are more clearly seen than in the profile of the DDCs ridge. To obtain the theoretical SDCs the elastic scattering cross section of figure 
4(a) has first to be folded with the Compton profile of the bound projectile electron. This leads to a damping and a slight shift of the Ramsauer-Townsend minima. This EIA calculation shows good agreement with the experimental data in the forward hemisphere except that it must not be used at $\theta_{f} \leqslant 20^{\circ}$ (since the EIA uses a final target scattering state it can never reproduce the cusp). For projectile electron emission in the backward hemisphere the deviations from theory are larger, even when the Dl contribution is included in the theory. As has been found for the comparable, but less asymmetric, collision system $\mathrm{H}^{0}(0.5 \mathrm{MeV}) \rightarrow \operatorname{Ar}(\mathrm{Kuzel}$ et al 1992) the doubly inelastic process gains importance at backward angles.

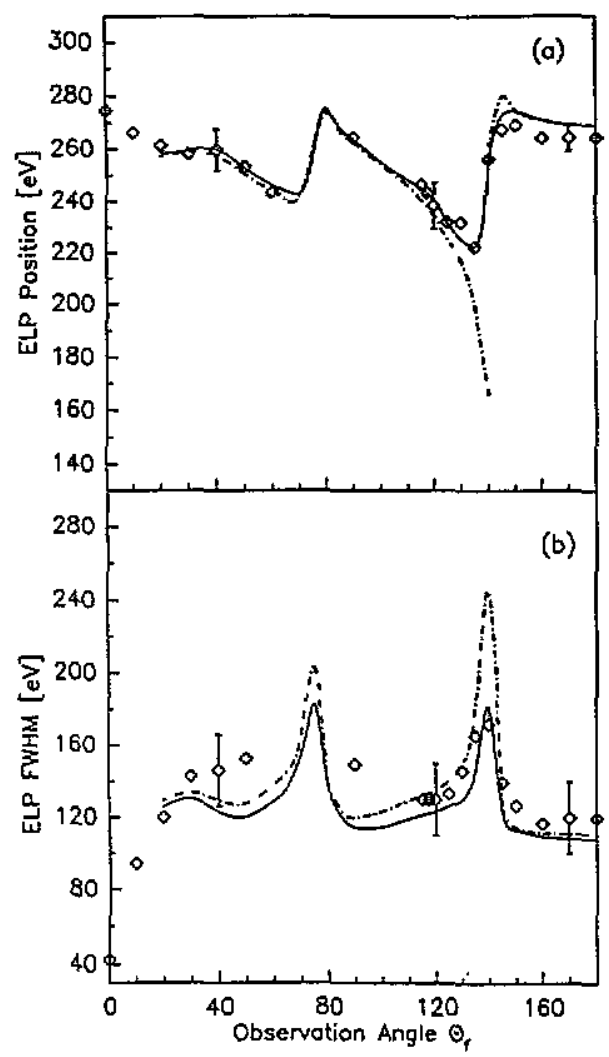

Figure 5. (a) Position of the electron loss peak maximum for $\mathrm{H}^{0} \rightarrow \mathrm{Kr}$. (b) FWHM of the electron loss peak: $\diamond$, experiment; $-\longrightarrow$, theory, EIA, sI; - - - theory, EIA, $\mathrm{SI}+\mathrm{DI}$.

In figures $5(a)$ and $(b)$ we show the position and the full width at half maximum (FWHM) of the ELP in the measured $\mathrm{Kr}$ spectra. At the angles associated with the Ramsauer-Townsend minima we see a shift of the peak position as large as $50 \mathrm{eV}$ and a strong increase of its width. Close to the forward direction the peak width becomes very small. This is due to electron loss into a continuum state of the projectile.

The si theory describes the variations in the peak position rather well. This is also true for the peak width at backward angles. However, the experimental FWHM at angles between $30^{\circ}$ and $90^{\circ}$ is underestimated by theory even when the DI contribution is included. The very strong variations in the peak position and width predicted by the DI theory-which even exhibits two peaks near $140^{\circ}$-are probably an artefact resulting 


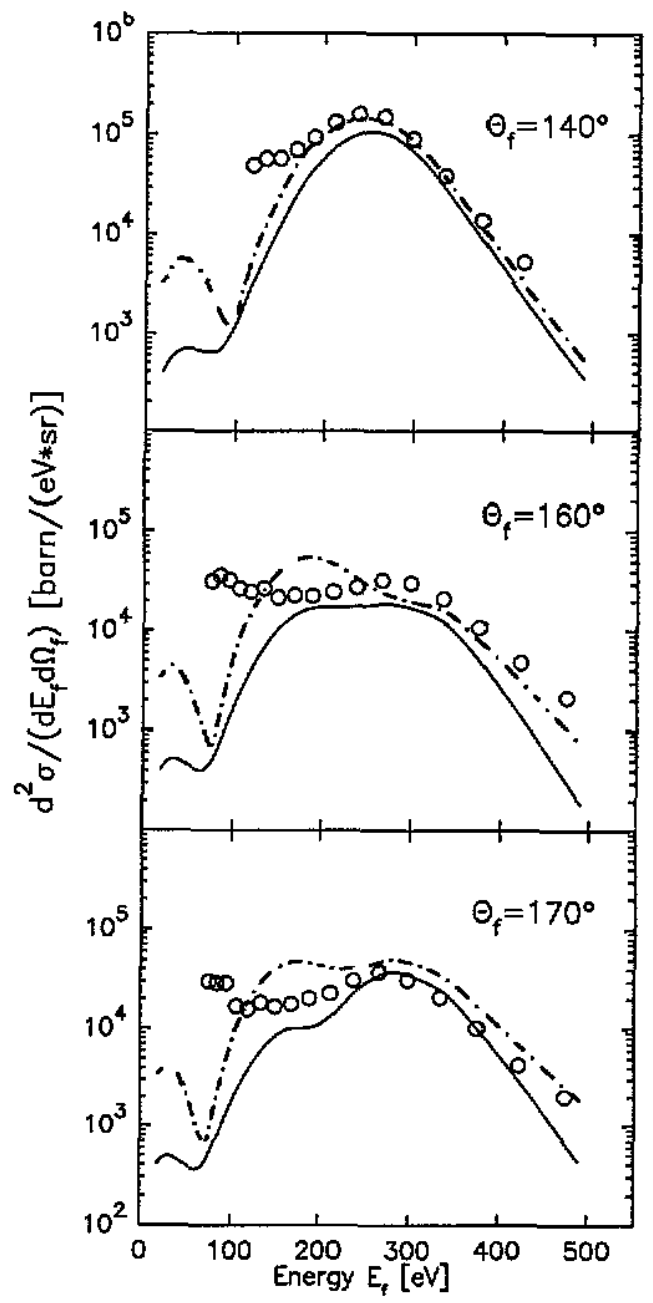

Figure 6. DDCs for $\mathrm{H}^{\circ} \rightarrow \mathrm{Xe}$, electron emission angles $\theta_{f}=140^{\circ}, 160^{\circ}$ and $170^{\circ}$. Legend as figure 3.

from the peaking approximation in (3.11). Without such an approximation the $D I$ structures are expected to be damped by the additional integration.

Additionally we measured absolute doubly differential cross sections $\left(\mathrm{d}^{2} \sigma / \mathrm{d} E_{f} \mathrm{~d} \Omega_{f}\right)$ for the more strongly asymmetric $\mathrm{H}^{0}(0.5 \mathrm{MeV}) \rightarrow \mathrm{Xe}$ system. In figure 6 we show three spectra for backward angles. The ELP at $140^{\circ}$ is well described by the sum of the $\mathrm{si}$ and DI contributions, but at $160^{\circ}$ and $170^{\circ}$ agreement only occurs over a limited range of energies. These two angles are particularly close to a predicted Ramsauer-Townsend minimum and it is possible that the $\mathrm{DI}$ part of the theory is failing here due to the peaking approximation.

Again we extracted singly differential cross sections $\left(\mathrm{d} \sigma / \mathrm{d} \Omega_{f}\right)$ by integrating over the range $150 \mathrm{eV}<E_{f}<400 \mathrm{eV}$. Figure 7 shows the SDCs, the peak position and peak width from collisions with this Xe target. To date we are only able to show the comparison between theory and experiment for the case of the sDCs. A proper comparison for peak position and FWHM requires better statistics for our DDCS at angles other than 




Figure 7. (a) SDCs for $\mathrm{Xe}$ target. $\mathrm{H}^{0}$ projectile: $\Delta$, experiment, DDCs integrated over the range $150<E_{f}<400 \mathrm{eV} ;-$, EIA, SI; - - - EIA, SI + DI, $e^{-}$elastic scattering at $300 \mathrm{eV}:---$, McCarthy et al (1977). (b) and (c), width and peak position. Legend as figure 5 .

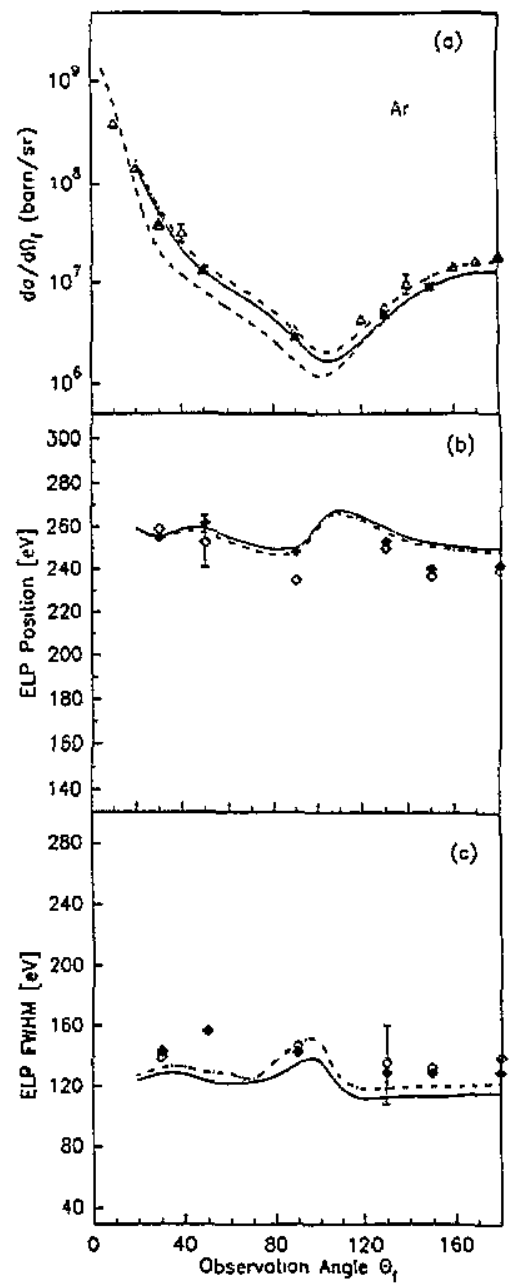

Figure 8. (a) SDCs, (b) electron loss peak position, (c) FWHM for an Ar target. The same symbols are used as in figure 7 but, in addition, coincidence data are shown as full symbols.

the three shown in figure 6(a). We see, however, that theory is now predicting three Ramsauer-Townsend minima together with their associated strong variations in peak position and width. Moreover, the inclusion of the DI channel is particularly important at angles greater than $50^{\circ}$ for this heavy target.

\section{Conclusion}

We have measured absolute doubly differential cross sections for electron emission occurring in strongly asymmetric collisions of the simplest electron carrying projectile $\mathrm{H}^{0}$. From these data we can extract singly differential cross sections for projectile 
ionization. When we compare the sDCs for the $\mathrm{Kr}$ and $\mathrm{Xe}$ targets (figures $4(b), 7(a)$ with that previously measured for the Ar target at the same collision velocity (figure $(8 a)$ ) we see that at forward angles the absolute cross sections are very similar for all systems. At larger angles, however, the SDCs does not vary smoothly with angle as it does for the light He target (Kövér et al 1988). In contrast it shows an increasing number of Ramsauer-Townsend minima as we pass down the periodic table. Associated with these minima are variations in the position and width of the electron loss peak. While for the Ar target these variations are rather modest (figure $8(b, c)$ ) - such that they could not be identified within the experimental error in earlier investigations (Kövér et al 1983, 1988) - they are very prominent for $\mathrm{Kr}$ and are predicted to be even more violent in the case of Xe.

The measurements and calculations presented in this work demonstrate that projectile ionization occurring in strongly asymmetric atom-atom collisions can be interpreted as an elastic scattering of 'quasi'-free electrons by the target potential. The electron impact approximation uses this as the frame for a quantum mechanical formalism, representing the 'quasi'-free character of the projectile electrons by the Compton profile. The features arising from this basic idea show convincing agreement of experimental and theoretical data even on an absolute scale. The inclusion of doubly inelastic processes is important, particularly for the heavy Xe target, although at backward angles our DI results may not be very accurate because of the peaking approximation involved. In principle, the precision of the present singles-mode experiments for $\mathrm{Kr}$ and $\mathrm{Xe}$ can be improved by recording the charge state of the transmitted projectile in coincidence with the emitted electrons. From the case of Ar where this has been done (figure 8) it is clear that a coincidence experiment will preserve the essential results of this new work.

\section{Acknowledgments}

This work has been funded by the 'Deutsche Forschungsgemeinschaft' under project number Gr 647/9, the British Council and the DAAD, and by GSI, Darmstadt.

\section{References}

Aashamar K, Luke T M and Talman J D 1978 At. Data Nucl. Data Tables 22443

Bates D R and Griffing G 1954 Proc Phys. Soc. A 67 663-8

Bernardi G, Suárez S, Focke P and Meckbach W 1988 Nucl. Instrum. Methods B 33 321-5

Bethe H A and Salpeter E E 1957 Quantum Mechanics of One and Two Electron Atoms (Berlin: Springer)

Burch D, Wieman H and Ingalls W B 1973 Phys. Rev. Lett. 30 823-6

Clementi E and Roetti C 1974 At. Data Nucl. Data Tables 14177

Cox H L and Bonham R A 1967 J. Chem. Phys. 47 2599-608

Crooks G B and Rudd M E 1970 Phys. Rev. Lett. 251599

Drepper F and Briggs J S 1976 J. Phys. B: At. Mol. Phys. 9 2063-71

DuBois R D and Manson S T 1990 Phys. Rev. A 421222

Hartley H M and Walters H R J 1987 J Phys. B:At. Mol. Phys. 203811

Jakubaßa D H 1980 J. Phys. B: At. Mol. Phys. 13 2099-108

Jakubaßa-Amundsen D H 1992 Z. Phys. D 22 701-11

1993 J. Phys. B. At. Mol. Opt. Phys. 26 L227-35

Kövér Á, Szabó Gy. Gulyás L, Tökési K, Berényi D, Heil O and Groeneveld K O 1988 J. Phys. B: At. Mol. Opt. Phys. 21 3231-41

Kövér Ȧ, Varga D, Szabó Gy, Berényi D, Kádár I, Ricz S, Végh J and Hock G $1983 \mathrm{~J}$. Phys. B: At. Mol. Phys. 16 1017-27 
Kuzel M, Heil O, Maier R, Lucas M W, Jakubaßa-Amundsen D H, Farmery B W and Groeneveld K O 1992 J. Phys. B: At. Mol. Opt. Ply's. 25 1839-51

Landau L D and Lifschitz E M 1970 Relativistische Quantentheorie vol 4a (Berlin: Akademie) \$37

McCarthy I E, Noble C J, Phillips B A and Turnbull A D 1977 Phys. Rev. A 15 2173-85

McEachran R P and Stauffer A D 1984 J. Phys. B: At. Mol. Plys. 17 2507-18

Miller T M and Bederson B 1977 Adv. At. Mol. Phys. 13 (see p 49)

Montenegro E C, Melo W S, Meyerhof W E and de Pinho A G 1992 Phys. Rev, Lett. 693033

Ramsauer C 1921 Ann. Phys., Lpz. 66546

Rudd M E, Toburen L $H$ and Stolterfoht N 1976 At. Data Nucl. Data Tables $23405-42$

Sienkiewicz J E and Baylis W E 1991 J. Phys. B: At. Mol. Opt. Phys. 24 265-74

Sin Fai Lam L T 1982 J. Phys. B: At. Mol. Phys. $15119-42$

Strand T G and Bonham R A 1964 J. Chem. Phys, 401686

Townsend J S and Bailey V A 1922 Phil. Mag, 43593

Wang J, Reinhold C O and Burgdörfer J 1992 Phys. Rev. A 454507

Wilson W E and Toburen L H 1973 Phys. Rev. A 71535 